\title{
Emergency department visits, ambulance calls, and mortality associated with an exceptional heat wave in Sydney, Australia, 2011: a time-series analysis
}

Andrea Schaffer ${ }^{1 *}$, David Muscatello ${ }^{1}$, Richard Broome², Stephen Corbett ${ }^{3}$ and Wayne Smith ${ }^{2}$

\begin{abstract}
Background: From January 30-February 6, 2011, New South Wales was affected by an exceptional heat wave, which broke numerous records. Near real-time Emergency Department (ED) and ambulance surveillance allowed rapid detection of an increase in the number of heat-related ED visits and ambulance calls during this period. The purpose of this study was to quantify the excess heat-related and all-cause ED visits and ambulance calls, and excess all-cause mortality, associated with the heat wave.
\end{abstract}

Methods: ED and ambulance data were obtained from surveillance and administrative databases, while mortality data were obtained from the state death registry. The observed counts were compared with the average counts from the same period from 2006/07 through 2009/10, and a Poisson regression model was constructed to calculate the number of excess ED visits, ambulance and deaths after adjusting for calendar and lag effects.

Results: During the heat wave there were 104 and 236 ED visits for heat effects and dehydration respectively, and 116 ambulance calls for heat exposure. From the regression model, all-cause ED visits increased by $2 \%$ (95\% Cl 1.01-1.03), all-cause ambulance calls increased by 14\% (95\% Cl 1.11-1.16), and all-cause mortality increased by 13\% (95\% Cl 1.06-1.22). Those aged 75 years and older had the highest excess rates of all outcomes.

Conclusions: The 2011 heat wave resulted in an increase in the number of ED visits and ambulance calls, especially in older persons, as well as an increase in all-cause mortality. Rapid surveillance systems provide markers of heat wave impacts that have fatal outcomes.

Keywords: Heat wave, Australia, Emergency department, Ambulance, Mortality, Syndromic surveillance

\section{Background}

Heat wave events can have considerable adverse impacts on morbidity and mortality. During an extreme heat wave in France in 2003, maximum daily temperatures exceeded $35^{\circ} \mathrm{C}$ for 15 days; the number of excess deaths across the country due to the heat wave was estimated to be 14,800 , equivalent to a $60 \%$ increase over the expected count [1]. In Paris alone, there were 2228 excess deaths with a 5 -fold increased risk of death observed on the hottest day of the heat wave [2].

\footnotetext{
* Correspondence: andrea.schaffer@sydney.edu.au

'Centre for Epidemiology and Research, New South Wales Department of Health, Sydney, Australia

Full list of author information is available at the end of the article
}

In Chicago in 1995 there was a 5-day heat wave which peaked on July 13 with a maximum temperature of $40^{\circ} \mathrm{C}$ [3]. During the month of July there were an estimated 696 excess deaths, equivalent to a $30 \%$ increase. As is often observed, the effect of the heat wave was delayed, with the greatest daily increase in mortality recorded two days after the hottest heat wave day, with an excess of 275 deaths [4]. Smaller increases in mortality during less severe heat waves have also been observed in Europe [5-9], China [10], and the United States [11,12].

It has been suggested that some of the excess deaths observed during heat waves are due to "mortality displacement", meaning that the deaths of patients who would have died in the coming days or weeks are shifted 
forward. Evidence from France and Chicago in 2003 and 1995 suggest that mortality displacement accounts for only a small percentage of the excess deaths [2,13], but results from other studies have been inconsistent [14].

Hospitalisations have also been found to increase during extreme heat events, especially among older persons [7,15-17], although this finding is not universal [18]. The effect of heat waves on emergency department visits and ambulance calls is less well established, but the existing evidence suggests that they also increase [17,19-21]. During an 18-day heat wave in 2006 in California an excess of 16,000 ED visits was observed, including 2134 excess visits for heat-related illness [20].

The elderly are most susceptible to the effects of extreme heat [22], due to physiological changes that occur with ageing, chronic illness, certain medications, and sedentary lifestyles which contribute to reduced body temperature regulation and dehydration [23]. Other vulnerable populations include those with cardiovascular [24], respiratory [6,25], or renal disease [25,26], and those with mental health problems $[27,28]$.

In Australia, although the more general association of increasing temperature and mortality has been studied [29], there are few studies that focus on the outcomes of specific heat wave events. A study from Adelaide of two extreme heat waves in 2008 and 2009 found an increase in ambulance calls of $10 \%$ and $16 \%$ respectively, and an increase in ED visits of 6\% only during the 2008 heat wave. There was no increase in mortality for all ages in either heat wave [17], while a study from Brisbane of a 20-day heat wave found an excess of 75 deaths [30].

New South Wales (NSW) is the most populous state in Australia with a population of over 7 million people [31], the majority of whom live near the coast, which has a temperate climate. From January 30 to February 6, 2011, there was an exceptional heat wave in NSW. In the Hunter Valley, there was a record-breaking consecutive 6 days with a maximum temperature above $39^{\circ} \mathrm{C}$. Overnight temperatures were similarly extreme, with Sydney experiencing 5 nights above $24^{\circ} \mathrm{C}$, including a record-breaking overnight temperature of $27.6^{\circ} \mathrm{C}$ on February 6 [32].

Syndromic surveillance of emergency departments (EDs) is a useful tool for public health monitoring [33-35], including during heat wave events [36,37]. The NSW Ministry of Health operates near real-time syndromic surveillance of ED presentations [38] and ambulance dispatches using automatic capture and analysis of electronic ED and ambulance data, and provides early warning of daily and weekly increases in disease activity in the population. During the NSW heat wave, statistically higher than expected increases in ED visits and ambulance calls were identified, both overall and for heat-related syndromes, which included heat stroke and dehydration. Thus, the purpose of the study was to quantify the number of excess ED visits and ambulance calls attributable to a period of exceptional heat in a temperate region of Australia, and to determine whether there was a concomitant increase in the number of deaths.

\section{Methods}

\section{Heat wave definition}

The period of January 30-February 6 was identified by the Bureau of Meteorology, Australia's national weather agency, as a period of exceptional heat in the Sydney and Hunter regions of NSW [32]. Using this independent heat wave definition offered an unbiased and objective way to define this heat wave prior to undertaking the analysis.

\section{Study area}

The study area was defined as the geographic region of NSW that experienced the maximum anomaly $\left(6^{\circ} \mathrm{C}\right)$ above the historical average (1961-1990) during the heat wave. Geographic boundaries matching the study area were identified from the Australian Standard Geographical Classification. This region included the Statistical Divisions of Sydney, Hunter and Illawarra, and the Statistical Local Areas of Mid-Western Regional Part B, Lithgow, Oberon, Goulburn Mulwaree (Goulburn) and Goulburn Mulwaree (Bal) and comprised $8 \%$ of the land area of NSW and 73\% of its population, i.e. 5.2 million people (Figure 1).

\section{Study period and data}

The study period was restricted to the southern hemisphere summer (November 1-February 28) to reduce seasonal effects. The daily maximum and minimum overnight temperatures were obtained from the Australian Bureau of Meteorology. Data from weather stations located within the heat wave region were averaged to provide the mean maximum/minimum temperatures for

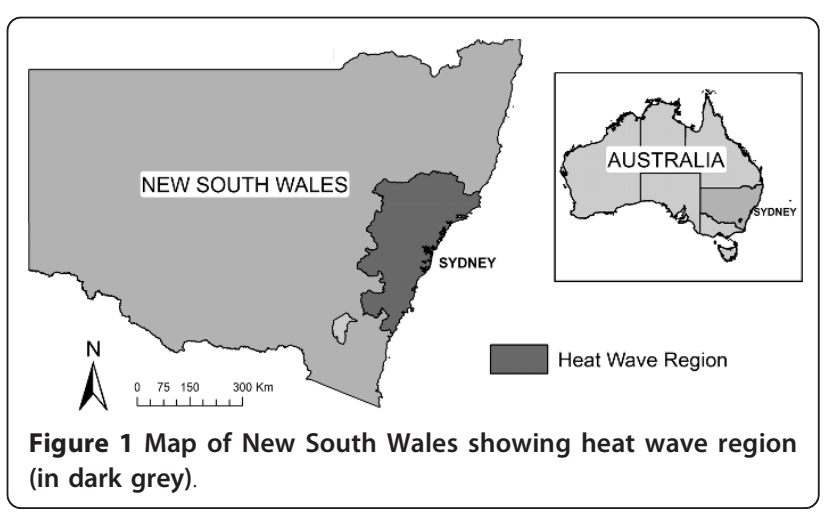


the heat wave region for each day. To provide a reference period, the daily maximum and minimum temperatures for the same weather stations for the summers from 2006/07 through to 2009/10 inclusive were averaged to give the mean daily maximum and minimum temperatures over the four years.

The ED and ambulance data were obtained from a combination of an ED-based syndromic surveillance system and the Emergency Department Data Collection, which contains routinely collected data captured in the ED clinical information systems of public hospitals across NSW. The ED data contain information on the patient's demographics, as well as their reason for presenting to the ED, including the triage nurse's notes. The ambulance data is a computerised database that captures information on all ambulance dispatches in response to persons who have called emergency services, including demographics and the reason for the call. Data from the 41 EDs within the heat wave region that participate in the syndromic surveillance were available, which account for $96 \%$ of all ED visits in this region. Ambulance data for the entire study period had only been collected for the Sydney Ambulance Division region and thus the analysis of ambulance data was restricted to this area, which services approximately $82 \%$ of the population in the heat wave region. February 12 and 13, 2011, were excluded from analysis of ambulance calls as data were incomplete on those dates.

Heat-related syndromes were classified using either the International Classification of Diseases, 10th Revision (ICD-10), the International Classification of Diseases, 9th Revision (ICD-9) or the Systematized Nomenclature of Medicine (SNOMED). Heat-related ED visits included individuals assigned a provisional diagnosis of: heatstroke, sunstroke, heat syncope, heat cramp, heat exhaustion, heat fatigue, heat oedema and other effects of heat (ICD-10-AM code T67, ICD-9-AM code 992, and equivalent SNOMED codes); and exposure to excessive natural heat (ICD-10-AM code X30, ICD-9-AM code E900.0 and equivalent SNOMED codes). ED visits for dehydration included a provisional diagnosis of volume depletion (ICD-10-AM code E86, ICD-9-AM code 276.5 and equivalent SNOMED codes). The list of SNOMED codes used is available in Table S1 (see Additional file 1: Table S1). Heat-related ambulance calls were assigned a problem category of heat or cold exposure. Since only data from the summer period were included it was assumed that all of the ambulance calls assigned this problem category were for heat exposure.

All-cause mortality data were obtained from the NSW Registry of Births, Deaths, and Marriages, which records all deaths that occur in the state, and includes information on the last known residential address of the deceased. Using this information the death data were geocoded and restricted to all persons who resided within the boundaries of the heat wave region at the time of their death. Cause-specific mortality was not available at the time of the study.

This study was approved by the NSW Population and Health Services Research Ethics Committee (CINSW LNR 2011/006).

\section{Statistical analysis}

To demonstrate the signals observed through the syndromic surveillance, a descriptive analysis was performed comparing total observed counts during the heat wave period to average observed counts from previous years for all-cause outcomes and for the more heat-specific syndromes used in the near real-time ED and ambulance surveillance system, i.e. ED visits for heatrelated conditions, dehydration, and ambulance calls for heat exposure. The average observed counts were calculated by averaging the counts for each day for the summer period from 2006/07 through to 2009/10 inclusive.

Between 2006 and 2010 the population of NSW has increased by $6 \%$, while the population over 75 years has increased by $8 \%$ and therefore ED visits, ambulance calls and deaths have been increasing over time. Thus, to control for this trend and other confounders a generalised linear model for count data, Poisson regression, was fit to determine the relative and absolute increase in the number of all-cause ED visits, all-cause ambulance calls, and all-cause deaths that were due to the heat wave. Data from the summer periods from November 1 to February 28 for the years 2006/07 through to 2010/11 inclusive were used in this analysis.

Variables for day of the week, public holidays, and quadratic variables for day of the year were included to control for calendar effects and any remaining seasonal effects and a variable for year was included to control for long term trends. An 8-day period representing the heat wave was modelled using an indicator variable set to 1 if the date fell between the start date and end date of this period, and 0 otherwise. A heat wave lag effect was considered, where the heat wave time period was shifted forward by $1,2,3$ or 4 days. The most appropriate lag for a given outcome was determined by which model resulted in the lowest Akaike Information Criterion. As studies have found that those aged over 75 years are more susceptible to heat effects [22], for each outcome 3 analyses were performed: one for those aged $<75$ years, one for those aged $\geq 75$ years, and one for all ages. The model fit was checked using deviance and residual plots. To check for mortality displacement the period following the heat wave was modelled to check for a reduction in the number of deaths. 


\section{Results}

From January 30 through February 6, the heat wave period, the average maximum temperature in the study area was $35.2^{\circ} \mathrm{C}$, and ranged from $32.0^{\circ} \mathrm{C}$ on January 30 to $38.5^{\circ} \mathrm{C}$ on February 5 . The average minimum overnight temperature was $21.8^{\circ} \mathrm{C}$ and reached a high of $23.7^{\circ} \mathrm{C}$ on February 5.

During the 8-day heat wave, there were 104 ED visits for heat effects and 236 ED visits for dehydration, and 116 ambulance calls for heat exposure (Table 1). The greatest daily counts for ED visits and ambulance calls for heat effects were observed on the hottest day of the heat wave (February 5), with 33 visits and 32 calls respectively. The highest daily count of ED visits for dehydration was observed the next day, with 45 visits. These were the highest daily counts observed in the past 5 years (Figure 2). All-cause outcomes were also higher, with 34,197 total ED visits, 8609 ambulance calls and 814 deaths during the heat wave period, as compared to an average of 30,092 visits, 7155 calls and 682 deaths in previous years, although the proportion of this increase that is due specifically to the heat wave is not clear.

From the regression modelling, the greatest relative increase in all-cause ED visits was observed with a lag of 3 days; after adjusting for calendar effects, there was a significant increase of $2 \%$ (RR 1.02, 95\% CI 1.01-1.03) which corresponded to an excess of 595 (95\% CI 221973) ED visits (Table 2). Similar results were observed in those under 75 years only (RR 1.01, 95\% CI 1.001.02), and in those over 75 years there was an increase of $8 \%$ (RR 1.08, 95\% CI 1.04-1.11).

The greatest relative increase in all-cause ambulance calls was observed with a lag of 1 day; after adjustment, there was a significant increase of $14 \%$ (RR 1.14, 95\% CI 1.11-1.16) corresponding to an excess of 1033 (95\% CI $845-1225)$ calls. In the younger age group a $10 \%$ increase in the number of ambulance calls was observed

\begin{tabular}{|c|c|c|}
\hline & Observed (2011) & Average observed (2007-2010) \\
\hline \multicolumn{3}{|l|}{ ED visits } \\
\hline All-cause & 34,197 & 30,092 \\
\hline Heat effects & 104 & 13.5 \\
\hline Dehydration & 236 & 81.3 \\
\hline \multicolumn{3}{|c|}{ Ambulance calls* } \\
\hline All-cause & 8609 & 7155 \\
\hline Heat exposure & 116 & 8.0 \\
\hline \multicolumn{3}{|l|}{ Deaths } \\
\hline All-cause & 814 & 682 \\
\hline
\end{tabular}

*Sydney only
(RR 1.10, 95\% CI 1.07-1.13) while in those over 75 years a $17 \%$ increase was observed (RR 1.17, 95\% CI 1.121.23).

In the adjusted model without any lag, there was a $13 \%$ increased risk of all-cause death (RR 1.13, 95\% CI 1.06-1.22). This corresponded to a predicted excess of 96 deaths (95\% CI 40-157). When stratified by age, in those aged less than 75 years there was a $16 \%$ increase in the number of deaths (RR 1.16, 95\% CI 1.02-1.30). In persons over 75 years an increase of $12 \%$ was observed (RR 1.12, 95\% CI 1.03-1.23).

\section{Discussion}

This is one of the few studies of the impact of a heat wave event on mortality, ambulance calls and ED visits in Australia. The strength of this current study is that it combines three sources of data to form a more complete picture of the effect of a specific heat wave event, and provides useful information on the timing of the peaks of the three outcomes, which can help in predicting the effects of future heat waves.

In this study, large numbers of ED visits and ambulance calls for heat-related conditions such as heat stroke, heat exhaustion and dehydration were observed during the heat wave, with the highest counts recorded in five years, emphasizing the exceptionality of this event. The effect of the heat wave was likely exacerbated by the fact that the hottest days occurred on the weekend, when it is likely that many people would be outdoors. The largest single daily counts of ED visits and ambulance calls for heat-related conditions were recorded on the Saturday during the heat wave. The absolute count of all cause ED visits and ambulance calls were also elevated in comparison to previous years, although this increase may have been partly due to a growing population and not specific to the heat wave, hence the need for multivariate analysis to eliminate the effect of long-term trends.

The Poisson model found significant increases for all outcomes and for both age groups. As expected, those aged 75 years or older experienced the highest excess rates of ED visits, ambulance calls and deaths (Table 2). This age group also experienced the largest absolute increase in the number of deaths, with an excess of 58 deaths predicted by the model to be attributable to the heat wave period, compared to 38 in younger persons, even though they represent a smaller population. In contrast, the largest absolute increases in ED visits and ambulance calls were observed in those aged less than 75 years, which is expected as they normally account for the majority of ED visits and ambulance calls $(86 \%$ and $63 \%$ respectively). Overall, the greatest absolute impact we found was on the number of ambulance calls with 1033 excess calls attributable to the heat wave, this 

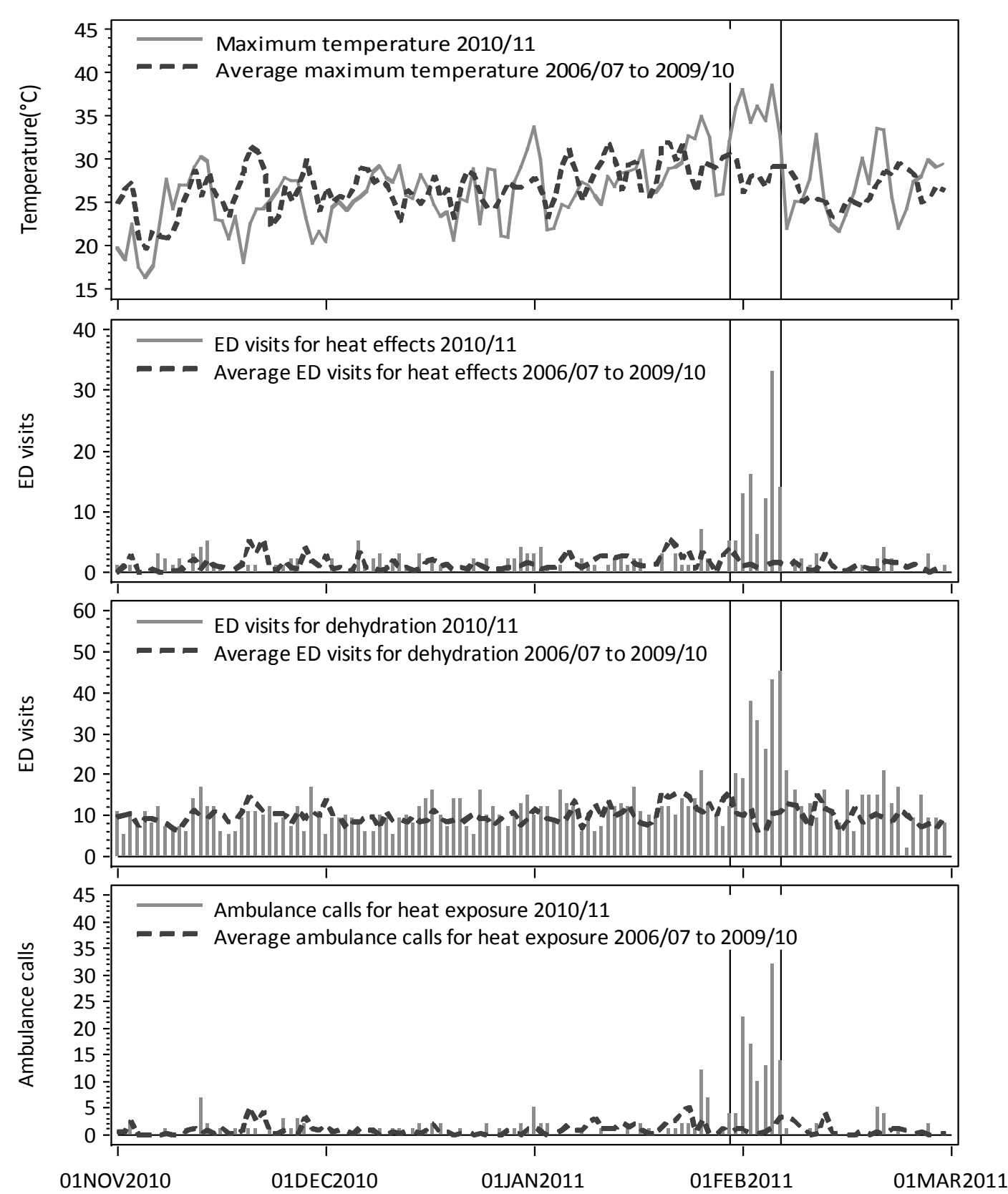

Figure 2 Daily temperature and daily counts of ED visits and ambulance calls for dehydration and heat-related conditions

despite the fact that the ambulance data only covered $82 \%$ of the population of the heat wave area. If ambulance data from the entire heat wave area been available, the number of excess calls would likely have been even greater.

While not as extreme as the events in France and Chicago, the impact of the heat wave on all-cause mortality overall that we observed was similar to that found in many northern hemisphere studies, despite the fact that heat effects are generally considered to be lower in regions with higher average temperatures [39], such as Australia. Although, in contrast to previous studies that have generally found a greater relative increase in mortality in older persons $[7,8]$, in the current study the increase in mortality was greater in persons younger than 75 years. This result is similar to those of one of the few other studies of heat waves in Australia. The Nitschke et al. [17] study from Adelaide of a 2009 heat wave also found a large increase in ambulance calls, but a smaller relative increase in ED visits. They also found 
Table 2 Relative risk and predicted excess counts and rates based on the model with the most appropriate lag for each outcome

\begin{tabular}{|c|c|c|c|}
\hline & $\begin{array}{l}\text { Relative Risk } \\
(95 \% \mathrm{Cl})\end{array}$ & $\begin{array}{l}\text { Predicted excess count } \\
(95 \% \mathrm{Cl})\end{array}$ & $\begin{array}{l}\text { Predicted excess rate per } \\
100,000 \text { population }(95 \% \mathrm{Cl})\end{array}$ \\
\hline \multicolumn{4}{|c|}{ All-cause ED visits* } \\
\hline All ages & $1.02(1.01-1.03)$ & $595(221-973)$ & $11.5(4.37-18.8)$ \\
\hline$<75$ years & $1.01(1.00-1.02)$ & $336(-9-685)$ & $6.9(-0.9-14.1)$ \\
\hline$\geq 75$ years & $1.08(1.04-1.11)$ & $322(186-462)$ & $100.0(57.8-143.5)$ \\
\hline \multicolumn{4}{|c|}{ All-cause ambulance calls (Sydney only)^ } \\
\hline All ages & $1.14(1.11-1.16)$ & $1033(845-1225)$ & $24.4(19.9-28.9)$ \\
\hline$<75$ years & $1.10(1.07-1.13)$ & $514(364-668)$ & $12.9(9.1-16.7)$ \\
\hline$\geq 75$ years & $1.17(1.12-1.23)$ & $323(229-421)$ & $135.8(96.3-177.1)$ \\
\hline \multicolumn{4}{|c|}{ All-cause deathst } \\
\hline All ages & $1.13(1.06-1.22)$ & $96(40-157)$ & $1.9(0.8-3.0)$ \\
\hline$<75$ years & $1.16(1.02-1.30)$ & $38(6-75)$ & $0.8(0.1-1.5)$ \\
\hline$\geq 75$ years & $1.12(1.03-1.23)$ & $58(13-107)$ & $18.0(4.0-33.2)$ \\
\hline
\end{tabular}

${ }^{*}$ Lag of 3 days; ${ }^{\wedge}$ Lag of 1 day; ${ }^{\dagger}$ No lag

a large relative increase in death in younger persons (1564 years), but in contrast they did not find any significantly increased mortality in older persons.

Unfortunately cause of death was not available at the time of the analysis and thus we were not able to investigate whether there were any cause-specific increases or to identify deaths that were potentially heat-related. But, given the wide-ranging health effects of extreme temperatures, all-cause mortality is the most sensitive measure of heat-related mortality. The period following the heat wave was modelled to check for mortality displacement. None was found, but this is difficult to assess as the effects could be obscured by subsequent hot days, as following the heat wave there were multiple days with maximum temperatures exceeding $30^{\circ} \mathrm{C}$.

While the 8 days of the heat wave clearly resulted in increased health care utilisation, the extreme heat may have only been one factor in the observed results. The effect of the high heat may have been modified by air pollution and/or high humidity, both of which are associated with increased morbidity and mortality [40-42]. Both high levels of ozone and above average humidity were experienced during the heat wave period $[32,43]$. As well, there were certainly other periods of extreme heat during the time period of the analysis. This may underestimate the effect of this heat wave in comparison to non-heat wave periods; but, the fact that we still found significant increases in all outcomes once again suggests that this event was exceptional.

The increases in the existing heat-related syndromes included in our regular syndromic surveillance reports were plausible given the clear heat exposure. Given that delays in death registrations arising from administrative processes, the syndromic ED and ambulance data available for analysis within 24 hours may be useful as a marker of potential mortality increases that are less easily and rapidly measured. Exploration of the predictive value of these syndromes for mortality outcomes would be valuable in the design of heat wave early warning systems.

\section{Conclusions}

This heat wave in NSW placed a great burden on the health care system. Not only was an increase in the number of heat-related ED visits and ambulance calls observed during this exceptional heat wave, but we also found a substantial impact on both all-cause ED visits and all-cause ambulance calls, especially in older populations who are known to be vulnerable to the effects of extreme heat. This occurred in tandem with an increase in the number of all-cause deaths.

\section{Additional material}

Additional file 1: Table S1. List of SNOMED codes used to identify ED visits for dehydration and heat-related syndromes.

\section{Abbreviations}

ED: Emergency department; NSW: New South Wales; ICD-10-AM: International Classification of Diseases; 10th Revision; Australian Modification; ICD-9-AM: International Classification of Diseases; 9th Revision; Australian Modification; SNOMED: Systematized Nomenclature of Medicine; $\mathrm{Cl}$ : Confidence interval.

\section{Acknowledgements}

We'd like to thank the New South Wales Department of Health, the New South Wales Biostatistical Officer Training Program and the Australian Bureau of Meteorology.

\section{Author details}

${ }^{1}$ Centre for Epidemiology and Research, New South Wales Department of Health, Sydney, Australia. 'Environmental Health Branch, New South Wales 
Department of Health, Sydney, Australia. ${ }^{3}$ Centre for Population Health, Sydney West Area Health Service, Sydney, Australia.

\section{Authors' contributions}

$\mathrm{DM}, \mathrm{RB}, \mathrm{SC}$ and WS conceived the study. AS contributed to the study design, conducted the analysis and drafted the manuscript. DM contributed to study design and assisted in the analysis. RB contributed to study design. All authors revised the manuscript and have read and approved the final manuscript.

\section{Competing interests}

The authors declare that they have no competing interests.

Received: 8 October 2011 Accepted: 24 January 2012 Published: 24 January 2012

\section{References}

1. Hémon D, Jougla E: Surmortalité liée à la canicule d'août 2003. Rapport d'étape (1-3). Estimation de la surmortalité et principales caractéristiques épidémiologiques. [http://www.cepidc.inserm.fr/inserm/html/pdf/ rapport_canicule_03.pdf].

2. Le Tertre A, Lefranc A, Eilstein D, Declercq C, Medina S, Blanchard M, Chardon B, Fabre P, Filleul L, Jusot JF, et al: Impact of the 2003 heatwave on all-cause mortality in 9 French cities. Epidemiology 2006, 17:75-79.

3. Centers for Disease Control and Prevention (CDC): Heat-related mortalityChicago, July 1995. MMWR Morb Mortal Wkly Rep 1995, 44:577-579.

4. Whitman S, Good G, Donoghue ER, Benbow N, Shou W, Mou S: Mortality in Chicago attributed to the July 1995 heat wave. Am J Public Health 1997, 87:1515-1518.

5. Rooney C, McMichael AJ, Kovats RS, Coleman MP: Excess mortality in England and Wales, and in Greater London, during the 1995 heatwave. J Epidemiol Community Health 1998, 52:482-486.

6. D'Ippoliti D, Michelozzi P, Marino C, De'Donato F, Menne B, Katsouyanni K, Kirchmayer U, Analitis A, Medina-Ramon M, Paldy A, et al: The impact of heat waves on mortality in 9 European cities: results from the EuroHEAT project. Environ Health 2010, 9:37-45.

7. Johnson H, Kovats RS, McGregor G, Stedman J, Gibbs M, Walton H, Cook L, Black E: The impact of the 2003 heat wave on mortality and hospital admissions in England. Health Stat Q 2005, 25:6-11.

8. Conti S, Meli P, Minelli G, Solimini R, Toccaceli V, Vichi M, Beltrano C, Perini L: Epidemiologic study of mortality during the Summer 2003 heat wave in Italy. Environ Res 2005, 98:390-399.

9. Hertel S, Le Tertre A, Jockel KH, Hoffmann B: Quantification of the heat wave effect on cause-specific mortality in Essen, Germany. Eur J Epidemiol 2009, 24:407-414.

10. Huang W, Kan H, Kovats S: The impact of the 2003 heat wave on mortality in Shanghai, China. Sci Total Environ 2010, 408:2418-2420.

11. Ostro BD, Roth LA, Green RS, Basu R: Estimating the mortality effect of the July 2006 California heat wave. Environ Res 2009, 109:614-619.

12. Anderson GB, Bell ML: Heat waves in the United States: mortality risk during heat waves and effect modification by heat wave characteristics in 43 U.S. communities. Environ Health Perspect 2011, 119:210-218.

13. Kaiser R, Le Tertre A, Schwartz J, Gotway CA, Daley WR, Rubin CH: The effect of the 1995 heat wave in Chicago on all-cause and cause-specific mortality. Am J Public Health 2007, 97(Suppl 1):S158-S162.

14. Martiello MA, Giacchi MV: High temperatures and health outcomes: a review of the literature. Scand J Public Health 2010, 38:826-837.

15. Mastrangelo G, Fedeli U, Visentin C, Milan G, Fadda E, Spolaore P: Pattern and determinants of hospitalization during heat waves: an ecologic study. BMC Public Health 2007, 7:200-207.

16. Semenza JC, McCullough JE, Flanders WD, McGeehin MA, Lumpkin JR: Excess hospital admissions during the July 1995 heat wave in Chicago. Am J Prev Med 1999, 16:269-277.

17. Nitschke M, Tucker GR, Hansen AL, Williams $S$, Zhang $Y$, Bi P: Impact of two recent extreme heat episodes on morbidity and mortality in Adelaide South Australia: a case-series analysis. Environ Health 2011, 10:42.

18. Kovats RS, Hajat S, Wilkinson P: Contrasting patterns of mortality and hospital admissions during hot weather and heat waves in Greater London, UK. Occup Environ Med 2004, 61:893-898.

19. Cerutti B, Tereanu C, Domenighetti G, Cantoni E, Gaia M, Bolgiani I, Lazzaro M, Cassis I: Temperature related mortality and ambulance service interventions during the heat waves of 2003 in Ticino (Switzerland). Soz Praventivmed 2006, 51:185-193.

20. Knowlton K, Rotkin-Ellman M, King G, Margolis HG, Smith D, Solomon G, Trent R, English P: The 2006 California heat wave: impacts on hospitalizations and emergency department visits. Environ Health Perspect 2009, 117:61-67.

21. Dhainaut JF, Claessens YE, Ginsburg C, Riou B: Unprecedented heat-related deaths during the 2003 heat wave in Paris: consequences on emergency departments. Crit Care 2004, 8:1-2.

22. Astrom DO, Forsberg B, Rocklov J: Heat wave impact on morbidity and mortality in the elderly population: a review of recent studies. Maturitas 2011, 69:99-105

23. Kenney WL, Munce TA: Invited review: aging and human temperature regulation. J Appl Physiol 2003, 95:2598-2603.

24. Bouchama A, Dehbi M, Mohamed G, Matthies F, Shoukri M, Menne B: Prognostic factors in heat wave related deaths: a meta-analysis. Arch Intern Med 2007, 167:2170-2176.

25. Conti S, Masocco M, Meli P, Minelli G, Palummeri E, Solimini R, Toccaceli V Vichi M: General and specific mortality among the elderly during the 2003 heat wave in Genoa (Italy). Environ Res 2007, 103:267-274.

26. Hansen AL, Bi P, Ryan P, Nitschke M, Pisaniello D, Tucker G: The effect of heat waves on hospital admissions for renal disease in a temperate city of Australia. Int J Epidemiol 2008, 37:1359-1365.

27. Hansen A, Bi P, Nitschke M, Ryan P, Pisaniello D, Tucker G: The effect of heat waves on mental health in a temperate Australian city. Environ Health Perspect 2008, 116:1369-1375.

28. Kaiser $\mathrm{R}$, Rubin $\mathrm{CH}$, Henderson AK, Wolfe Ml, Kieszak S, Parrott CL Adcock M: Heat-related death and mental illness during the 1999 Cincinnati heat wave. Am J Forensic Med Pathol 2001, 22:303-307.

29. Bi P, Williams S, Loughnan M, Lloyd G, Hansen A, Kjellstrom T, Dear K, Saniotis A: The effects of extreme heat on human mortality and morbidity in Australia: implications for public health. Asia Pac J Public Health 2011, 23:27S-36S.

30. Tong S, Ren C, Becker N: Excess deaths during the 2004 heatwave in Brisbane, Australia. Int J Biometeorol 2010, 54:393-400.

31. Australian Bureau of Statistics (ABS): Population by Age and Sex, Regions of Australia, 2009. [http://www.abs.gov.au/ausstats/abs@.nsf/Products/ 3235.0 2009 Main+Features New+South+Wales].

32. Bureau of Meteorology (BOM): An exceptional summer heatwave in greater Sydney and the Hunter Valley. Special Climate Statement 27. [http://www.bom.gov.au/climate/current/statements/scs27.pdf].

33. Heffernan R, Mostashari F, Das D, Karpati A, Kulldorff M, Weiss D: Syndromic surveillance in public health practice, New York City. Emerg Infect Dis 2004, 10:858-864

34. Marx MA, Rodriguez CV, Greenko J, Das D, Heffernan R, Karpati AM, Mostashari F, Balter S, Layton M, Weiss D: Diarrheal illness detected through syndromic surveillance after a massive power outage: New York City, August 2003. Am J Public Health 2006, 96:547-553.

35. Yuan CM, Love S, Wilson M: Syndromic surveillance at hospital emergency departments-southeastern Virginia. MMWR Morb Mortal Wkly Rep 2004, 53(Suppl):56-58.

36. Josseran L, Fouillet A, Caillere N, Brun-Ney D, llef D, Brucker G, Medeiros H, Astagneau P: Assessment of a syndromic surveillance system based on morbidity data: results from the Oscour network during a heat wave. PLoS One 2010, 5:e11984-e11991.

37. Leonardi GS, Hajat S, Kovats RS, Smith GE, Cooper D, Gerard E: Syndromic surveillance use to detect the early effects of heat-waves: an analysis of NHS direct data in England. Soz Praventivmed 2006, 51:194-201.

38. Muscatello DJ, Churches T, Kaldor J, Zheng W, Chiu C, Correll P, Jorm L: An automated, broad-based, near real-time public health surveillance system using presentations to hospital Emergency Departments in New South Wales, Australia. BMC Public Health 2005, 5:141-152.

39. Anderson BG, Bell ML: Weather-related mortality: how heat, cold, and heat waves affect mortality in the United States. Epidemiology 2009, 20:205-213.

40. Filleul L, Cassadou S, Medina S, Fabres P, Lefranc A, Eilstein D, Le Tertre A, Pascal L, Chardon B, Blanchard $M$, et al: The relation between temperature, ozone, and mortality in nine French cities during the heat wave of 2003. Environ Health Perspect 2006, 114:1344-1347. 
41. Hu W, Mengersen K, McMichael A, Tong S: Temperature, air pollution and total mortality during summers in Sydney, 1994-2004. Int I Biometeorol 2008, 52:689-696.

42. Lin S, Luo M, Walker RJ, Liu X, Hwang SA, Chinery R: Extreme high temperatures and hospital admissions for respiratory and cardiovascular diseases. Epidemiology 2009, 20:738-746.

43. New South Wales Goverment Office of Environment and Heritage: Air quality data.[http://www.environment.nsw.gov.au/AQMS/index.htm].

doi:10.1186/1476-069X-11-3

Cite this article as: Schaffer et al: Emergency department visits, ambulance calls, and mortality associated with an exceptional heat wave in Sydney, Australia, 2011: a time-series analysis. Environmental Health 2012 11:3.

Submit your next manuscript to BioMed Central and take full advantage of:

- Convenient online submission

- Thorough peer review

- No space constraints or color figure charges

- Immediate publication on acceptance

- Inclusion in PubMed, CAS, Scopus and Google Scholar

- Research which is freely available for redistribution

Submit your manuscript at www.biomedcentral.com/submit 